\section{PCAST provides assessment of US Nanotechnology Initiative}

www.nano.gov role in nearly every technology-based sector of society.

The US National Nanotechnology Initiative (NNI) is a crosscutting national vision for nanotechnology research and development (R\&D) in the United States. Established in 2001, the NNI is not directly funded and has no centralized budget or management, but is rather an initiative involving 20 federal in nanotechnology can play an important

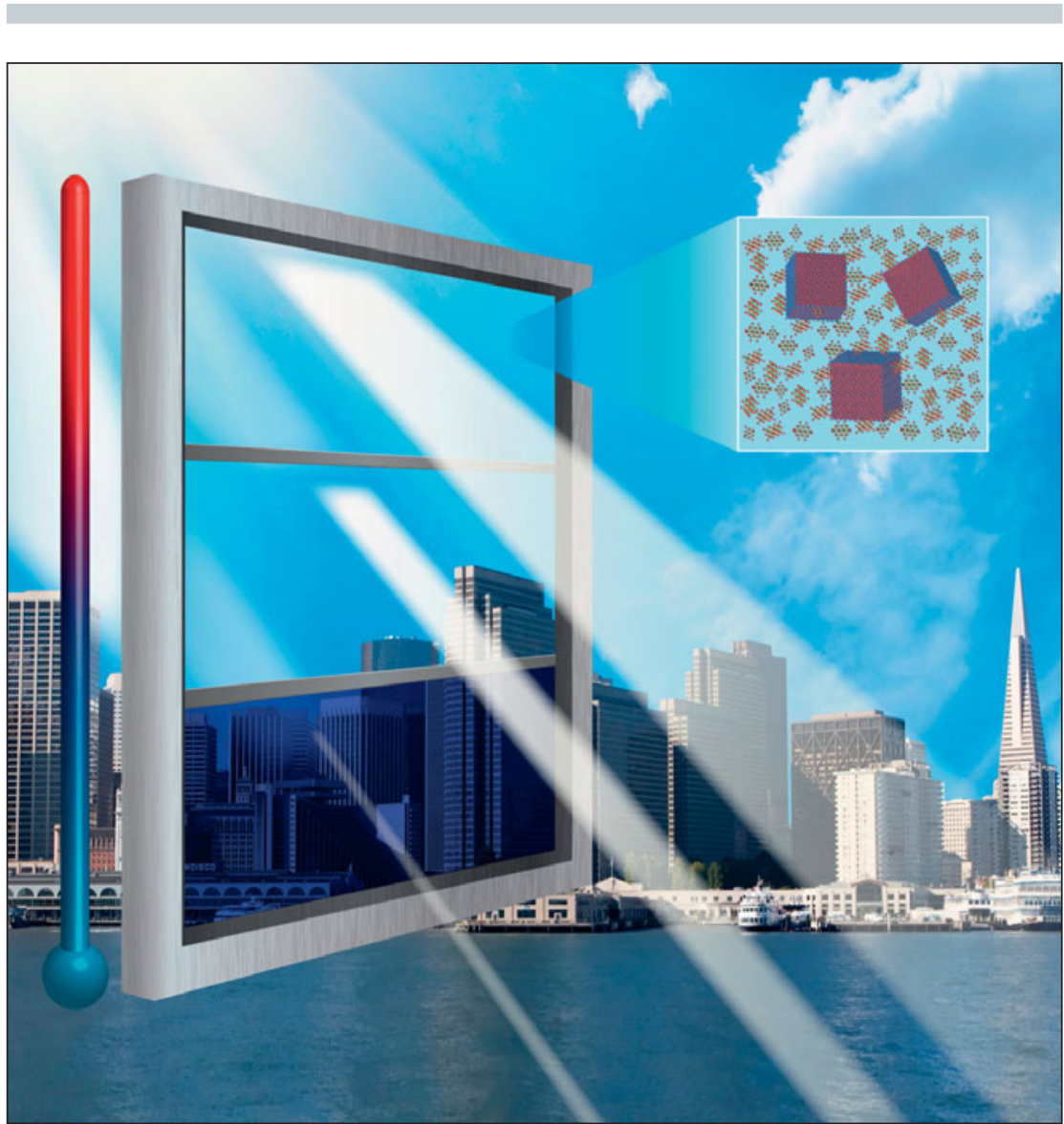

Graphic shows the "smart window" technology developed at the Molecular Foundry at Lawrence Berkeley National Laboratory as part of the US National Nanotechnology Initiative. These windows employ switchable electrochromic materials - tin-doped indium oxide nanocrystals in a niobium oxide matrix (shown in the inset) - that absorb different light and heat frequencies. The bright mode, which allows both light and heat through, is shown on top of the window. The cool mode, which blocks most of the heat but allows light through, is shown in the middle. And the dark mode, which blocks both heat and light, is shown at the bottom. Credit: Anna Llordés, Delia Milliron and Creative Services, Lawrence Berkeley National Lab.

departments, independent agencies, and independent commissions working together to revolutionize nanotechnology across the range of industries to the benefit of society.

Coordinated by the Nanoscale Science, Engineering, and Technology Subcommittee of the National Science and Technology Council Committee on Technology and the National Nanotechnology Coordination Office (NNCO), the NNI is reviewed biennially by a National Nanotechnology Advisory Panel as mandated in the 21st Century Nanotechnology R\&D Act of 2003. The President's Council of Advisors on Science and Technology (PCAST) was designated to fill this role through Executive Order 13539 in 2004, and has now assessed the NNI five times.

The fifth assessment of the NNI was reported to the President and Congress in October 2014. The assessment report characterizes the NNI as a "truly successful venture" stating that the investment of over \$20 billion in nanotechnology research and development (R\&D) over the duration of the initiative has led to "great success in creating the building blocks of nanoscience." The report cites two recent examples of NNI successes: the development of "smart glass" that can alter the transmission of heat and light through the glass surface, and the proofof-concept demonstration of carbon nanotubes that degrade a class of chemical nerve agents and can be incorporated into fabrics to produce protective clothing.

The NNCO also provided a number of examples of successful NNI technology developments including carbon nanotube sheets, yarns, powders, and arrays for aerospace, defense, aviation, automotive, energy, and consumer products; nano-based medicines and technologies to fight diseases and deliver targeted therapy; quantum dots for consumer electronics (primarily TV displays) and solar panels; and nano-based high-performance coatings with uses in machining, manufacturing, lubrication, textiles, biomedical, energy, and military applications.

"The first decade of the NNI drove significant breakthroughs in the efficient synthesis of nano-sized building 
blocks-nanowires, nanotubes, quantum dots, DNA, etc.," said Jeff Neaton, Director of the Molecular Foundry at Lawrence Berkeley National Laboratory. The Molecular Foundry is one of five Department of Energy user facilities created in response to the NNI where scientists from academia, government, and industry can collaborate on nanoscience projects. "Given the diversity of what can be made, the next challenge involves understanding and controlling the functionality that emerges from combining these different nano-sized building blocks," said Neaton.

In addition to highlighting the successes of the NNI, the assessment also lays out 12 recommendations spanning five categories - Grand Challenges; Program Management; Commercialization; Research Enterprise; and Environmental, Health, and Safety. Michael Meador, NNCO Director, said, "We feel that this was a very thoughtful review of the NNI by PCAST and they've gone out of their way to really understand how it functions and make recommendations that make good sense. We are already looking at how some of these recommendations might be implemented."

Michael McQuade, a PCAST member and $\mathrm{Co}-\mathrm{Chair}$ for the 5 th Assessment (along with Mark Gorenberg), articulated the success and potential for growth within the NNI, saying, "The initiative has shown excellent technology and science development to date and we've reached a point in the initiative where the opportunities for commercialization are better than they've ever been." McQuade emphasized the importance of not losing focus on basic nanoscience research, but also described one of the report's key recommendations as the need to "bring a new focus to commercialization of the technologies that are being developed as part of the initiative."

Meador agreed, saying, "The NNI needs to continue to do what it's already done well-R\&D, training, supporting user facilities, etc., but now there also needs to be more focus on commercialization." The assessment's primary recommendation to drive nanotechnology commercialization is to use the Grand Challenges framework. A Grand Challenge exhibits many characteristics - it must achieve an audacious but measurable goal with significant societal impact, it must establish a partnership between the public and private sectors, it must have clear interim milestones, and it must be broadly cross-disciplinary spanning from $\mathrm{R} \& \mathrm{D}$ to commercialization.

"Since this framework is already widely used in the science community and across the federal government, it is an ideal vehicle to bring nanotechnologies to market," said McQuade. Collaboration between the public and private sectors has already started in many areas of nanotechnology, and Neaton characterized the Molecular Foundry's engagement with industry as "strong and growing" and added that they already have a "strong history of successful partnerships." But Neaton also acknowledges that one of the biggest challenges going forward is "getting the word out about what capabilities are available and streamlining how industry can work with us to meet their scientific needs."

Lloyd Whitman, Assistant Director for Nanotechnology at the White House Office of Science and Technology Policy, says it's important for scientists to spread the word: "The NNI is not just an activity of the federal government; for the NNI to succeed, we need the public to understand the promise of nanotechnology-enabled products, and our students to be prepared to make those products possible."

\section{Jennifer A. Nekuda Malik}

ISTIC-TWAS report depicts high-impact innovation studies http://twas.org cooperation in science, technology, and innovation, in developing countries. TWAS - The World Academy of Sciences - is located in Italy and works toward advancing science and engineering for sustainable prosperity in the developing world.

Their book describes cases from 15 countries where innovation proved to be the keystone that turns challenges into opportunities for development, whether of new products or services.

For example, researchers from Redeemer's University in Nigeria have formed a composite adsorbent of defatted papaya seeds with locally sourced kaolinite clay. The composite demonstrates excellent adsorbent properties for lead and cadmium as well as for nickel ions, polycyclic aromatic hydrocarbons, and polychlorinated biphenyls found in water.

More than 100 candidates made up an initial list of innovation projects. Twenty-three of them were then selected on the basis of their replicability, impact, and the lessons they provide. They were also chosen for a presentation at a workshop held in Penang, Malaysia, in October 2012.

Now these cases have been grouped in a publication that ISTIC and TWAS will distribute among policymakers and other leaders to illustrate why investment in innovation is a critical part of effective governance. 